\title{
Sobrepeso e obesidade em universitários: prevalências e fatores associados
}

\section{Overweight and obesity in college students: prevalence and associated factors}

\section{AUTORES \\ Gerleison Ribeiro Barros ${ }^{1}$ (D) \\ Sueyla Ferreira da Silva dos Santos ${ }^{2}$ (D) \\ Alynne Christian Ribeiro Andaki ${ }^{1}$ (D) \\ Thiago Ferreira de Sousa ${ }^{1,3}$ (D) \\ 1 Universidade Federal do Triângulo Mineiro, Programa de Pós-Graduação em Educação Física, Uberaba, Minas Gerais, Brasil. \\ 2 Universidade Federal do Amazonas, Instituto de Ciências Sociais, Educacão e Zootecnia de Parintins, Amazonas, Brasil \\ 3 Universidade Federal do Recôncavo da Bahia, Centro de Formação de Professores, Amargosa, Bahia, Brasil.}

\section{CONTATO}

\section{Gerleison Ribeiro Barros}

efgerleison@hotmail.com

Av. Tutunas, nº490 - Bairro Tutunas, Ubera-

ba, Minas Gerais, Brasil.

CEP: $3806-500$.

DOI

$10.12820 /$ rbafs. $26 \mathrm{e} 0225$

\section{(cc) BY}

Este trabalho está licenciado com uma Licença Creative Commons - Atribuição 4.0 Internacional.

\begin{abstract}
RESUMO
O objetivo deste estudo foi estimar entre homens e mulheres universitários de Minas Gerais, as prevalências de sobrepeso e obesidade e suas associações com atividade física e tempo sentado. Este estudo transversal estimou por meio das medidas referidas da massa corporal e estatura, o índice de massa corporal (IMC) e analisou o sobrepeso (IMC: 25,0 a $29,9 \mathrm{~kg} / \mathrm{m}^{2}$ ) e a obesidade (IMC: $\geq 30,0$ $\mathrm{kg} / \mathrm{m}^{2}$ ). As variáveis independentes foram a atividade física e o tempo sentado, e as variáveis de controle foram sociodemográficas, de vínculo com a universidade e comportamentais, analisadas em três modelos. As associações foram estimadas via Odds Ratio (OR) por meio da Regressão Logística Multinomial. O nível de significância foi de 5\%. Participaram 1.105 universitários, sendo 61,6\% de mulheres. A média etária foi de 21,25 (desvio padrão $=4,1$ ) anos para as mulheres e para os homens foi de 21,83 (desvio padrão = 4,2) anos. As prevalências de sobrepeso e obesidade foram, respectivamente, $27,5 \%$ e $6,9 \%$ em homens e $15,5 \%$ e $8,0 \%$ em mulheres. O tempo sentado associou-se à obesidade em homens, independente apenas das características sociodemográficas (OR = 3,54; IC95\%: $1,04-12,12)$ e dos atributos sociodemográficos mais as características de vínculo com a universidade $(\mathrm{OR}=3,48$; IC95\%: 1,01 - 11,99). Com o ajuste para os comportamentos alimentares não houve associação (OR = 3,49; IC95\%: 0,99 - 12,23). Para as mulheres não foram observadas associações significativas. Conclui-se que a prevalência de sobrepeso foi elevada em homens. Os homens universitários com elevado tempo sentado apresentaram mais chances de serem obesos.
\end{abstract}

Palavras-chave: Índice de massa corporal; Sobrepeso; Obesidade; Estudantes.

\section{ABSTRACT}

The aim of this study was to estimate the prevalence of overweight and obesity and their associations with physical activity and sitting time among men and women university students in Minas Gerais, Brazil. This is a cross-sectional study that estimated the outcomes of overweight (BMI: 25.0 to $29.9 \mathrm{~kg} / \mathrm{m}^{2}$ ) and obesity (BMI: $\geq 30.0 \mathrm{~kg} / \mathrm{m}^{2}$ ) using the body mass index (BMI) by self-reported body mass and stature. The independent variables were physical activity and sitting time, and control variables were sociodemographic, link with the university and behavioral variables were included in three models. Association analyzes were performed via Odds Ratio (OR) using Multinomial Logistic Regression. The significance level was 5\%. A total of 1,105 university students participated in the study, comprising $61.6 \%$ of women. The mean age was of $21.25(S D=4.1)$ and $21.83(S D=4.2)$ for women and men, respectively. The prevalence of overweight and obesity in men was $27.5 \%$ and $6.9 \%$ and in women $15.5 \%$ and $8.0 \%$, respectively. Time sitting was associated with obesity in men, regardless of sociodemographic characteristics (OR $=3.54$; 95\%CI: 1.04 12.12) and university stage plus sociodemographic characteristics (OR $=3.48 ; 95 \% C I: 1.01-11.99)$. With the adjustment of health behaviors, the association was not observed (OR $=3.49 ; 95 \%$ CI: $0.99-12.23)$. For women, no significant associations were found. In conclusion, overweight prevalence was high in men. The university men with sitting time higher represented more chances of obesity.

Keywords: Body mass index; Overweight; Obesity; Students.

\section{Introdução}

O Brasil possui, segundo o Instituto Nacional de Estudos e Pesquisas Educacionais Anísio Teixeira (INEP) pouco mais de 8 milhões de universitários ${ }^{1}$. A transição da educação básica para a vida universitária surge como desafio, devido as mudanças desfavoráveis nos comportamentos de saúde e possivelmente contribuem para o ganho de peso $^{2}$, e com esse aumento favorece o risco de sobrepeso e obesidade ${ }^{3}$.
O índice de massa corporal (IMC) elevado pode maximizar e/ou desempenhar um papel secundário na ocorrência de morte por todas as causas ${ }^{4}$. E o sobrepeso, estimado pelo IMC, associa-se diretamente com fatores de risco cardiovasculares, como hipertensão arterial, dislipidemia e disglicemia, e pode ocasionar problemas futuros como a obesidade ${ }^{5}$. A obesidade e suas repercussões representam uma condição de riscos à saúde que ocasiona impacto na qualidade de vida, as- 
sociando-se a múltiplas comorbidades, como diabetes do tipo II, doenças cardiovasculares, síndrome da hipoventilação da obesidade, doença renal crônica, doença hepática e diferentes tipos de câncer, o que pode ter influência na expectativa de vida ${ }^{6}$.

Alguns fatores podem contribuir para o aumento de peso, dentre eles estão o tempo elevado em comportamento sedentário ${ }^{7}$ e a prática de atividade física em níveis insuficientes ${ }^{2}$. O comportamento sedentário corresponde às condutas de vigília caracterizadas por um gasto energético $\leq 1,5 \mathrm{MET}$, como por exemplo, nas posições sentada, reclinada ou deitada ${ }^{8}$. Por outro lado, aqueles que não atendem as diretrizes de atividade física, ou seja, pelo menos 150 minutos em intensidade moderada ou 75 minutos em intensidade vigorosa por semana, ou a combinação equivalente de ambas as intensidades, são considerados insuficientemente ativos ${ }^{9}$.

Em relação ao comportamento sedentário, observou-se que os universitários com elevado tempo nesta conduta apresentam mais chances de desenvolvimento de sobrepeso e obesidade 7 . Em relação à atividade física, estudo com universitários, por meio de monitoramento, foi observado o aumento das prevalências de excesso de peso em universitárias não praticantes de atividades físicas no lazer ${ }^{3}$. Estima-se que o comportamento sedentário em nível excessivo e atividade física em nível insuficiente associam-se com chances entre $95 \%$ a $168 \%$ de ocorrência de obesidade em adultos jovens ${ }^{10}$.

Deste modo, este estudo fundamenta-se na ocorrência do aumento das prevalências de sobrepeso e obesidade entre os universitários ${ }^{3}$ e que fatores comportamentais, como a atividade física e o comportamento sedentário, como o elevado tempo sentado, podem estar associados à presença destas condições nesse grupo, com importantes divergências entre homens e mulheres. Acredita-se que ser estudante vinculado a uma universidade possibilita riscos para o acometimento de sobrepeso e obesidade, em virtude das mudanças nos hábitos, como aqueles relacionados ao aumento da alimentação com elevado teor de açúcar e o consumo de bebidas alcoólicas².

As informações apresentadas neste estudo poderão ser úteis para a implementação de possíveis intervenções, por meio de projetos e programas institucionais, com foco na saúde do universitário, especificamente à manutenção do peso corporal. Soma-se a isso a contribuição para o estado da arte sobre o tema, quanto à caracterização das associações diretas das práticas de atividades físicas e tempo sentado sobre o IMC de uni- versitários. Diante disso, o objetivo deste estudo foi estimar entre homens e mulheres universitários de Minas Gerais, as prevalências e as associações entre a atividade física e o tempo sentado em relação ao sobrepeso e a obesidade.

\section{Método}

Estudo de delineamento transversal, derivado da coleta de dados da pesquisa "Perfil do estilo de vida e qualidade de vida dos estudantes da Universidade Federal do Triângulo Mineiro (UFTM)", aprovado pelo Comitê de Ética em Pesquisa (CEP) da instituição com o número de parecer: 2.402.734.

A população-alvo foram os universitários dos cursos presenciais, matriculados em todos os semestres dos cursos da UFTM $(\mathrm{N}=5.952)$, sede Uberaba, Minas Gerais, nos turnos diurno e noturno. Foi utilizada para a determinação do tamanho da amostra a equação de Luiz e Magnanini ${ }^{11}$. A prevalência empregada foi de $50 \%$, nível de confiança de $95 \%$ e erro aceitável de amostragem de três pontos percentuais, acrescido de $20 \%$ para perdas e mais $10 \%$ para controle dos fatores de confusão em estudos de associação. A amostra estimada foi de 1.195 universitários.

A amostra foi estratificada de forma proporcional à distribuição dos universitários em cada um dos 25 cursos de graduação presenciais da UFTM. Foi prevista a reposição das recusas. Foram considerados como critérios de inclusão, os universitários com 18 anos ou mais, independentes do sexo e condições físicas. Foram excluídos os universitários dos cursos de ensino a distância, com matrícula especial (estudantes com diploma de ensino superior que se matriculam em disciplinas de cursos de graduação), dos cursos técnicos e aqueles dos cursos de graduação pertencentes ao Campus de Iturama (Agronomia, Licenciatura em Ciências Biológicas e Química), pois apresentava menor tempo de implantação (ano 2015) e estrutura ainda divergente da sede, após a tabulação das informações. Tais ações foram possíveis em virtude das perguntas inseridas no instrumento, para fins de controle da amostra participante.

Adotou-se a forma de participação por conveniência e o recrutamento dos participantes ocorreu nas salas de aula da instituição, mediante a verificação da ocorrência de aulas na grade de horários dos cursos, visando garantir local em diferentes dias da semana. As coletas foram realizadas de forma individual ou em grupo de até 30 universitários nos meses de abril a julho de 2018 . Antes do início da coleta de dados realizou-se o trei- 
namento da equipe composta por 11 universitários e estudantes da pós-graduação em Educação Física da UFTM, no mês de março de 2018, de modo padronizar o processo de aplicação do instrumento e orientações sobre o estudo.

A coleta de dados foi realizada por meio do autopreenchimento de um questionário com duração média de 15 minutos, composto por perguntas do questionário Indicadores de Saúde e Qualidade de Vida de Acadêmicos (ISAQ-A) ${ }^{12}$, validado para universitários brasileiros e o International Physical Activity Questionnaire versão curta (IPAQ $)^{13}$, validado para pesquisas com adultos ${ }^{13}$.

A variável dependente foi o IMC e os desfechos foram o sobrepeso $\left(\geq 25 \mathrm{~kg} / \mathrm{m}^{2}\right.$ a $\left.29,9 \mathrm{~kg} / \mathrm{m}^{2}\right)$ e a obesidade $\left(\geq 30 \mathrm{~kg} / \mathrm{m}^{2}\right)$, calculada com base nas medidas autorreferidas da massa corporal e estatura, mediante a equação padrão (massa corporal dividida pela estatura ao quadrado $)^{14}$. A categoria de referência foi o IMC até $24,9 \mathrm{~kg} / \mathrm{m}^{2}$. O uso das medidas referidas da massa corporal e estatura, apresentam níveis satisfatórios de reprodutibilidade (estatura em metros e massa corporal em quilogramas, mediante a análise do gráfico de dispersão de Bland-Altman, com diferença média de -0,002, IC95\%: -0,006 a 0,003, e diferença média de 0,36, IC95\%: -0,19 a 0,91, respectivamente) $)^{12}$ e de validade concorrente com níveis aceitáveis de sensibilidade e especificidade ${ }^{15}$, para o emprego em pesquisas com universitários $^{12,15}$.

Como variáveis independentes o tempo sentado e a prática de atividades físicas, ambos mensurados pelo IPAQ ${ }^{13}$. O tempo sentado foi estimado considerando um dia de segunda-feira a sexta-feira, e em um dia de final de semana, sendo calculada a média ponderada do tempo sentado em um dia da semana, posteriormente classificado em 6 horas/dia ou mais e $<6$ horas $/ \mathrm{dia}^{16}$. A medida do tempo sentado do IPAQ apresenta nível de validade por meio da correlação de Spearman (rho) de $=0,79$ em estudo com adultos brasileiros ${ }^{13}$, e validade concorrente satisfatória em universitários brasileiros por meio do coeficiente de correlação intraclasse (CCI) para o tempo na semana $(\mathrm{CCI}=0,31)$ e final de semana $(\mathrm{CCI}=0,53)^{17}$.

Em relação à prática de atividade física, considerou-se a soma dos tempos destinados à caminhada, atividades de intensidade moderada e em intensidade vigorosa por semana e empregou-se a seguinte classificação: insuficientemente ativos (até 149 minutos) e ativos (150 minutos ou mais) conforme recomendação de prática para a população adulta brasileira ${ }^{9}$. Os minutos da prática em intensidade vigorosa foram multiplicados por dois na composição do tempo ${ }^{18}$. A medida de atividade física do IPAQ apresenta validade satisfatória $($ rho $=0,75)$ para o emprego em pesquisa com adultos ${ }^{13}$.

Para fins de análise das capacidades psicométricas das medidas das variáveis independentes em relação à amostra de universitários deste estudo, estimou-se a qualidade do ajustamento dos itens das variáveis independentes de forma quantitativa, atividade física (caminhada, atividade de intensidade moderada e intensidade vigorosa) e tempo sentado (tempo sentado durante um dia da semana e um dia do final de semana), por meio da análise fatorial confirmatória via software AMOS, versão 24.0. Considerou-se os seguintes índices e valores adequados ${ }^{19}$ : valor de $\mathrm{p}$ do qui-quadrado $\left(\chi^{2}\right)$ não significativo $(p>0,05)$; razão entre $\chi^{2}$ pelos graus de liberdade (df), com valores menores que dois; Comparative Fit Index (CFI), Normalized Fit Indices (NFI) e Tuker-Lewis Indices (TLI), valores acima de 0,90; Root Mean Square Error of Approximation (RMSEA) e intervalo de confiança a 90\% (IC90\%), os valores menores que 0,06 e Standardized Root Mean-Square Residual (SRMR), menor que 0,08. Os valores observados foram: $\mathrm{p}$ do $\chi^{2}=0,213 ; \chi^{2} / \mathrm{df}=1,421$; CFI $=0,995 ; \mathrm{NFI}=0,984 ; \mathrm{TLI}=0,990 ; \mathrm{RMSEA}=0,022$ (IC90\%: 0,001-0,055); SRMR = 0,02.

As variáveis descritivas e de controle foram selecionadas considerando o que a literatura apresenta quanto potenciais fatores de risco e proteção em relação à condição de excesso de peso em universitários ${ }^{2,3,20}$, sendo idade (anos completos) e situação conjugal (com parceiro e sem parceiro) como variáveis sociodemográficas; de vínculo com universidade, o tempo de universidade em anos (até 2 anos e 3 anos ou mais) e período de estudo (diurno e noturno); as variáveis comportamentais foram, o consumo de frutas (irregular: até 4 dias por semana; regular: 5 ou mais dias por semana $)^{21}$; o consumo de verduras (irregular: até 4 dias por semana; regular: 5 ou mais dias por semana) ${ }^{21}$; o consumo de carne vermelha gordurosa (nenhum dia e 1 ou mais dias por semana ${ }^{22}$; o consumo de frango com pele, sem remover a gordura visível (nenhum dia e 1 ou mais dias por semana) ${ }^{22}$; o consumo de salgados (nenhum dia e 1 ou mais dias por semana) $)^{22}$; o consumo de refrigerantes ou sucos artificiais (até 4 dias por semana e 5 ou mais dias por semana ${ }^{22}$; o consumo de bebidas alcoólicas em uma ocasião nos últimos 30 dias, sendo cinco doses ou mais para os homens e quatro doses ou mais para as mulheres (não e sim) ${ }^{23}$. 
As informações foram tabuladas no Excel, versão 2013. As análises para o atendimento ao objetivo deste estudo foram realizadas no Statistical Package for the Social Sciences (SPSS), versão 24.0. Foram empregadas análises das frequências absolutas e relativas, média e desvio padrão (DP). As análises de associação entre as variáveis independentes e os desfechos foram estimadas via Odds Ratio (OR) complementadas pelo Intervalo de Confiança a 95\% (IC95\%), por meio da Regressão Logística Multinomial nas análises brutas e ajustadas.

Para a realização das análises ajustadas, empregou-se inicialmente a estimativa de colinearidade entre as variáveis de controle e independentes do estudo. Foi utilizada a análise de correlação Tetrachoric (T), estimada por meio do software Stata 11.0 e considerou-se como aceitáveis os valores de correlação < 0,70 e $\mathrm{p}<0,05$ nas análises bivariadas. Os maiores valores de correlação observados foram para os homens entre o consumo de frango e consumo de carnes $(\mathrm{T}=0,628)$, e para as mulheres entre o consumo de verduras e consumo de frutas $(T=0,592)$. Diante disso, nenhuma variável foi excluída de forma prévia ao emprego das análises ajustadas.

Nas análises ajustadas entre a atividade física e o tempo sentado em relação aos desfechos, foi empregado o método de seleção de variáveis backward das variáveis de controle, que permaneceram no ajuste no mesmo modelo e seguiram não sendo mais removidas para o ajuste dos próximos modelos, aquelas com valor de $\mathrm{p}<0,20$ do teste de likelihood ratio. Foram adotados três modelos na análise ajustada, sendo no modelo I ajustado para as variáveis sociodemográficas, no modelo II ajustado para as variáveis de vínculo com a universidade mais as variáveis que permaneceram no ajuste no modelo I, e no modelo III, houve o ajuste para as variáveis comportamentais mais as variáveis que permaneceram nos ajustes nos modelos I e II. Ao analisar a atividade física como variável independente, o tempo sentado foi incluído como variável de ajuste no modelo III e da mesma forma a atividade física foi incluída no ajuste no modelo III, quando o tempo sentado foi a variável independente. Em ambas as situações essas variáveis seguiram o critério para a permanência no ajuste com o valor de $\mathrm{p}<0,20$.

Todas as análises foram realizadas separadas por sexo, em virtude das diferenças entre as prevalências de sobrepeso e obesidade entre os homens e as mulheres $\left(\chi^{2}=23,281, p=<0,01\right)$. Considerou-se o nível de significância de $5 \%$ nas análises.

\section{Resultados}

Participaram do estudo 1.159 universitários. Trinta e seis universitários, distribuídos entre os cursos não foram encontrados, mesmo alternando dias e horários de aulas, possivelmente por realizarem atividades em outros espaços da instituição como laboratórios, bem como em outros locais como nos estágios. Não houve perdas, ou seja, universitários que informaram o interesse em participar da pesquisa em dia e horário agendado e que não teriam sido encontrados para efetivar a participação. Após a coleta de dados, houve a exclusão de três participantes por idade inferior a 18 anos e 46 por portar diploma de ensino superior, perfazendo a amostra de 1.110 universitários (taxa de resposta de 96,99\%). Essa amostra não difere estatisticamente da população-alvo por curso (dados não apresentados).

Neste estudo, houve a participação de 422 homens e 683 mulheres, sendo que cinco universitários não informaram o sexo. A média etária entre os homens foi de 21,83 (DP = 4,2) anos e variou de 18 a 54 anos e entre as mulheres a média etária foi de $21,25(\mathrm{DP}=4,1)$ anos e variou de 18 a 56 anos. A Tabela 1 apresenta a descrição da amostra de universitários. Observou-se, entre as mulheres, que a maioria estava sem parceiro, com menor tempo de universidade e do período de estudo diurno. Entre os homens, a maioria sem parceiro, com maior tempo de universidade e do período diurno. Em relação à amostra geral, observou-se que $73,8 \%$ dos universitários estudavam no turno diurno. Destaca-se em todos os universitários o consumo de carne vermelha e de álcool em excesso.

Sobre o comportamento alimentar, notou-se a ocorrência tanto entre os homens, quanto entre as mulheres, elevadas prevalências de consumo irregular de frutas $(77,8 \%$ vs $73,7 \%$, respectivamente), consumo de carne vermelha $(92,8 \%$ vs $87,8 \%$, respectivamente) e consumo excessivo de álcool ( $71,7 \%$ vs $66,4 \%$, respectivamente). Observou-se que $27,7 \%$ dos universitários não atenderam as recomendações nacionais de atividade física e percebeu-se que as mulheres apresentaram predomínio de prática insuficiente de atividade física. Quanto ao tempo sentado, cerca de 75,9\% dos universitários passavam seis horas ou mais nesse comportamento.

Na Figura 1 é apresentada as prevalências de sobrepeso e obesidade de todos os universitários. Observou-se maiores prevalências de sobrepeso em homens do que em mulheres $(p<0,05)$. 
Tabela 1 - Distribuição da amostra por sexo e total, considerando as variáveis sociodemográficas, de vínculo com a universidade e comportamentais relacionados à saúde em universitários $(n=1.105)$. Uberaba, Minas Gerais. 2018.

\begin{tabular}{|c|c|c|c|c|c|c|}
\hline \multirow{2}{*}{ Variável } & \multicolumn{2}{|c|}{ Homens } & \multicolumn{2}{|c|}{ Mulheres } & \multicolumn{2}{|c|}{ Todos } \\
\hline & $\mathrm{n}$ & $\%$ & $\mathrm{n}$ & $\%$ & $\mathrm{n}$ & $\%$ \\
\hline \multicolumn{7}{|l|}{ Situação conjugal } \\
\hline Com companheiro & 12 & 2,8 & 30 & 4,4 & 43 & 3,9 \\
\hline Sem companheiro & 410 & 97,2 & 653 & 95,6 & 1.067 & 96,1 \\
\hline \multicolumn{7}{|l|}{ Tempo de curso } \\
\hline Até 02 anos & 201 & 47,9 & 362 & 53,1 & 566 & 51,1 \\
\hline 03 anos ou mais & 219 & 52,1 & 320 & 46,9 & 541 & 48,9 \\
\hline \multicolumn{7}{|l|}{ Período de estudo } \\
\hline Noturno & 98 & 23,3 & 189 & 27,7 & 291 & 26,2 \\
\hline Diurno & 323 & 76,7 & 494 & 72,3 & 818 & 73,8 \\
\hline \multicolumn{7}{|l|}{ Consumo de frutas } \\
\hline Irregular & 326 & 77,8 & 502 & 73,7 & 830 & 75,1 \\
\hline Regular & 93 & 22,2 & 179 & 26,3 & 275 & 24,9 \\
\hline \multicolumn{7}{|l|}{ Consumo de verduras } \\
\hline Irregular & 255 & 60,7 & 332 & 48,8 & 589 & 53,3 \\
\hline Regular & 165 & 39,3 & 348 & 51,2 & 516 & 46,7 \\
\hline \multicolumn{7}{|l|}{ Consumo de carne vermelha } \\
\hline Nenhum & 30 & 7,2 & 82 & 12,2 & 114 & 10,4 \\
\hline 01 dia ou mais & 388 & 92,8 & 591 & 87,8 & 982 & 89,6 \\
\hline \multicolumn{7}{|l|}{ Consumo de frango com pele } \\
\hline Nenhum & 123 & 29,2 & 330 & 48,6 & 457 & 41,4 \\
\hline 01 dia ou mais & 298 & 70,8 & 349 & 51,4 & 648 & 58,6 \\
\hline \multicolumn{7}{|l|}{ Consumo de salgados } \\
\hline Nenhum & 60 & 14,2 & 95 & 14,0 & 156 & 14,2 \\
\hline 01 dia ou mais & 359 & 85,1 & 582 & 86,0 & 945 & 85,8 \\
\hline \multicolumn{7}{|l|}{ Consumo de refrigerantes } \\
\hline Até 04 dias & 321 & 76,1 & 553 & 81,2 & 877 & 79,2 \\
\hline 05 dias ou mais & 101 & 23,9 & 128 & 18,8 & 231 & 20,8 \\
\hline \multicolumn{7}{|l|}{ Consumo excessivo de álcool } \\
\hline Não & 119 & 28,3 & 229 & 33,6 & 348 & 31,4 \\
\hline $\operatorname{Sim}$ & 302 & 71,7 & 452 & 66,4 & 759 & 68,6 \\
\hline \multicolumn{7}{|l|}{ Atividade física } \\
\hline Ativos & 324 & 77,9 & 464 & 68,9 & 791 & 72,3 \\
\hline Insuficientemente ativos & 92 & 22,1 & 209 & 31,1 & 303 & 27,7 \\
\hline \multicolumn{7}{|l|}{ Tempo sentado } \\
\hline$\geq 06$ horas & 298 & 71,5 & 531 & 78,4 & 834 & 75,9 \\
\hline$<06$ horas & 119 & 28,5 & 146 & 21,6 & 265 & 24,1 \\
\hline
\end{tabular}

$\mathrm{n}=\operatorname{amostra} ; \%=$ proporção.

As associações entre o tempo sentado em relação ao sobrepeso e a obesidade em homens são apresentadas na Tabela 2. Na análise bruta, verificou-se associação em relação à obesidade $(\mathrm{OR}=3,48$; IC95\%: 1,02

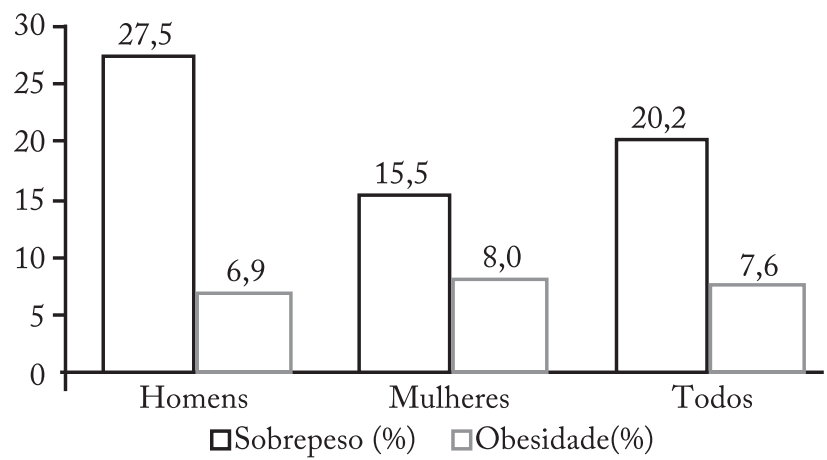

Figura 1 - Prevalência de sobrepeso e obesidade em universitários por sexo e total. Uberaba, Minas Gerais. 2018.

- 11,85), e houve a permanência da associação com o ajuste para as características sociodemográficas e de vínculo com a universidade, porém, quando controlado para o consumo de carne vermelha com gordura e o consumo de álcool a associação não foi observada. Para as mulheres, nos modelos I, II, III, não houve associação do tempo sentado em relação ao sobrepeso e a obesidade. Na Tabela 3, nas análises brutas e ajustadas não houve associação entre atividade física em relação ao sobrepeso e a obesidade em universitários, tanto em homens quanto em mulheres.

\section{Discussão}

Neste estudo observou-se que a prevalência de sobrepeso foi mais elevada entre os homens em comparação as mulheres. No estudo realizado com universitários do Kuwait, as prevalências de sobrepeso e obesidade foram maiores em homens quando comparado às mulheres $(28,7 \% \text { e } 23,8 \% \text { vs. } 19,9 \% \text { e } 12,1 \% \text {, respectivamente })^{24}$. $\mathrm{Na}$ Bahia, mediante inquéritos realizados com universitários em diferentes anos, em homens, o excesso de peso passou de 30,1\% em 2010 para 36,4\% em 2014 e em mulheres não foram encontradas diferenças significativas ${ }^{3}$. Possíveis explicações para o resultado deste estudo, podem estar relacionadas as questões alimen$\operatorname{tares}^{24}$ e de autoavaliação da imagem corporal ${ }^{25}$. Apesar de que ambos os sexos não se alimentam de forma apropriada, os homens tendem a apresentar mais comportamentos alimentares inadequados ${ }^{24}$. Destaca-se que os aspectos sociais, quanto ao estabelecimento das relações nesse período de formação profissional são relevantes estimuladoras quanto ao fortalecimento da valorização das atitudes relacionadas à estética ${ }^{24} \mathrm{e}$ favorecimento de condutas alimentares adequadas, especialmente em mulheres ${ }^{3,24}$, de forma que podem estimular percepções da imagem corporal, seja com o 
Tabela 2 - Associação entre o tempo sentado e o sobrepeso e a obesidade em universitários. Odds Ratio estimados via Regressão Logística Multinomial separadas por sexo. Uberaba, Minas Gerais. 2018.

\begin{tabular}{|c|c|c|c|c|c|c|c|c|}
\hline \multirow{4}{*}{ Variável } & \multirow{2}{*}{\multicolumn{2}{|c|}{ Análise bruta }} & \multicolumn{2}{|c|}{ Análise ajustada } & \multicolumn{2}{|c|}{ Análise ajustada } & \multicolumn{2}{|c|}{ Análise ajustada } \\
\hline & & & \multicolumn{2}{|c|}{ Modelo I* } & \multicolumn{2}{|c|}{ Modelo II** } & \multicolumn{2}{|c|}{ Modelo III"*** } \\
\hline & \multicolumn{2}{|c|}{ OR (IC95\%) } & \multicolumn{2}{|c|}{ OR (IC95\%) } & \multicolumn{2}{|c|}{ OR (IC95\%) } & \multicolumn{2}{|c|}{ OR (IC95\%) } \\
\hline & Sob. & Ob. & Sob. & Ob. & Sob. & $\mathrm{Ob}$. & Sob. & Ob. \\
\hline & \multicolumn{8}{|c|}{ Homens } \\
\hline$\geq 6$ horas & 1 & 1 & 1 & 1 & 1 & 1 & 1 & 1 \\
\hline$<6$ horas & $0,94(0,59-1,51)$ & $3,48(1,02-11,85)$ & $1,01(0,62-1,64)$ & $3,54(1,04-12,12)$ & $0,99(0,61-1,60)$ & $3,48(1,01-11,99)$ & $1,03(0,63-1,68)$ & $3,49(0,99-12,23)$ \\
\hline \multirow{5}{*}{ Variável } & \multirow{2}{*}{\multicolumn{2}{|c|}{ Análise bruta }} & \multicolumn{2}{|c|}{ Análise ajustada } & \multicolumn{2}{|c|}{ Análise ajustada } & \multicolumn{2}{|c|}{ Análise ajustada } \\
\hline & & & \multicolumn{2}{|c|}{ Modelo I } & \multicolumn{2}{|c|}{ Modelo II\# } & \multicolumn{2}{|c|}{ Modelo III\#\# } \\
\hline & \multicolumn{2}{|c|}{ OR (IC95\%) } & \multicolumn{2}{|c|}{ OR (IC95\%) } & \multicolumn{2}{|c|}{ OR (IC95\%) } & \multicolumn{2}{|c|}{ OR (IC95\%) } \\
\hline & Sob. & $\mathrm{Ob}$. & Sob. & $\mathrm{Ob}$. & Sob. & $\mathrm{Ob}$. & Sob. & $\mathrm{Ob}$. \\
\hline & \multicolumn{8}{|c|}{ Mulheres } \\
\hline$\geq 6$ horas & 1 & 1 & 1 & 1 & 1 & 1 & 1 & 1 \\
\hline$<6$ horas & $0,62(0,38-1,00)$ & $0,65(0,34-1,24)$ & $0,72(0,43-1,19)$ & $0,75(0,38-1,49)$ & $0,74(0,45-1,23)$ & $0,80(0,40-1,58)$ & $0,74(0,45-1,23)$ & $0,79(0,40-1,57)$ \\
\hline
\end{tabular}

OR = Odds ratio; IC95\% = Intervalo de Confiança a 95\%; Sob. = Sobrepeso; Ob. = Obesidade; *Modelo I, ajustado para: idade; **Modelo II, ajustado para as variáveis que permaneceram no ajuste no Modelo I mais: tempo de universidade; *** Modelo III, ajustado para variáveis que permaneceram nos ajustes nos Modelos I e II mais: consumo de carne vermelha e consumo de álcool; ¥Modelo I, ajustado para: idade; \#\#odelo II, ajustado para as variáveis que permaneceram no ajuste no Modelo I mais: tempo de universidade; \#Modelo III, ajustado para variáveis que permaneceram nos ajustes nos Modelos I e II mais: consumo de refrigerantes ou sucos; Valores de OR e IC95\% em negrito apresentaram associação com valor de $\mathrm{p}$ do teste de Wald $<0,05$.

Tabela 3 - Associação entre a atividade física e o sobrepeso e a obesidade em universitários. Odds Ratio estimados via Regressão Logística Multinomial separadas por sexo. Uberaba, Minas Gerais. 2018.

\begin{tabular}{|c|c|c|c|c|c|c|c|c|}
\hline \multirow{4}{*}{ Variável } & \multirow{2}{*}{\multicolumn{2}{|c|}{ Análise bruta }} & \multicolumn{2}{|c|}{ Análise ajustada } & \multicolumn{2}{|c|}{ Análise ajustada } & \multicolumn{2}{|c|}{ Análise ajustada } \\
\hline & & & \multicolumn{2}{|c|}{ Modelo I* } & \multicolumn{2}{|c|}{ Modelo II** } & \multicolumn{2}{|c|}{ Modelo III*** } \\
\hline & \multicolumn{2}{|c|}{ OR (IC95\%) } & \multicolumn{2}{|c|}{ OR (IC95\%) } & \multicolumn{2}{|c|}{ OR (IC95\%) } & \multicolumn{2}{|c|}{ OR (IC95\%) } \\
\hline & Sob. & $\mathrm{Ob}$. & Sob. & Ob. & Sob. & Ob. & Sob. & Ob. \\
\hline & \multicolumn{8}{|c|}{ Homens } \\
\hline$\overline{\text { Ativos }}$ & 1 & 1 & 1 & 1 & 1 & 1 & 1 & 1 \\
\hline $\begin{array}{l}\text { Insuficientemente } \\
\text { ativos }\end{array}$ & $\begin{array}{c}0,94(0,55- \\
1,61)\end{array}$ & $\begin{array}{c}1,71(0,73- \\
3,98)\end{array}$ & $\begin{array}{c}0,88(0,51 \text { - } \\
1,52)\end{array}$ & $\begin{array}{c}1,70(0,73- \\
3,97)\end{array}$ & $\begin{array}{c}0,86(0,50- \\
1,50)\end{array}$ & $\begin{array}{c}1,49(0,62- \\
3,60)\end{array}$ & $\begin{array}{c}0,93(0,53- \\
1,62)\end{array}$ & $\begin{array}{c}1,42(0,58- \\
3,50)\end{array}$ \\
\hline \multirow{5}{*}{ Variável } & \multirow{2}{*}{\multicolumn{2}{|c|}{ Análise bruta }} & \multicolumn{2}{|c|}{ Análise ajustada } & \multicolumn{2}{|c|}{ Análise ajustada } & \multicolumn{2}{|c|}{ Análise ajustada } \\
\hline & & & \multicolumn{2}{|c|}{ Modelo I\# } & \multicolumn{2}{|c|}{ Modelo II\#\# } & \multicolumn{2}{|c|}{ Modelo III\#\# } \\
\hline & \multicolumn{2}{|c|}{ OR (IC95\%) } & \multicolumn{2}{|c|}{ OR (IC95\%) } & \multicolumn{2}{|c|}{ OR (IC95\%) } & \multicolumn{2}{|c|}{ OR (IC95\%) } \\
\hline & Sob. & Ob. & Sob. & $\mathrm{Ob}$. & Sob. & Ob. & Sob. & Ob. \\
\hline & \multicolumn{8}{|c|}{ Mulheres } \\
\hline Ativos & 1 & 1 & 1 & 1 & 1 & 1 & 1 & 1 \\
\hline $\begin{array}{l}\text { Insuficientemente } \\
\text { ativos }\end{array}$ & $\begin{array}{c}1,50(0,96- \\
2,33)\end{array}$ & $\begin{array}{c}0,98(0,52- \\
1,84)\end{array}$ & $\begin{array}{c}1,46(0,93- \\
2,29)\end{array}$ & $\begin{array}{c}0,92(0,48- \\
1,75)\end{array}$ & $\begin{array}{c}1,41(0,90- \\
2,22)\end{array}$ & $\begin{array}{c}0,83(0,43- \\
1,61)\end{array}$ & $\begin{array}{c}1,41(0,90 \text { - } \\
2,22)\end{array}$ & $\begin{array}{c}0,83(0,43- \\
1,61)\end{array}$ \\
\hline
\end{tabular}

OR = Odds ratio; IC95\% = Intervalo de Confiança a 95\%; Sob. = Sobrepeso; Ob. = Obesidade; *Modelo I, ajustado para: idade; **Modelo II, ajustado para as variáveis que permaneceram no ajuste no Modelo I mais: tempo de universidade; ****Modelo III, ajustado para variáveis que permaneceram nos ajustes nos Modelos I e II mais: consumo de carne vermelha, consumo de álcool e tempo sentado; $\neq$ Modelo I, ajustado para: idade; \#Modelo II, ajustado para as variáveis que permaneceram no ajuste no Modelo I mais: tempo de universidade; \#\#Modelo III, ajustado para variáveis que permaneceram nos ajustes nos Modelos I e II mais: não houve variáveis comportamentais ajustadas; Valores de OR e IC95\% em negrito apresentaram associação com valor de $\mathrm{p}$ do teste de Wald <0,05.

desejo de aumentar ou diminuir a silhueta corporal ${ }^{25}$, e assim possibilitam a manutenção do peso corporal.

Neste estudo, a prevalência do tempo sentado em excesso foi elevada, além disso, foi associada com chances três vezes maiores de apresentar obesidade em homens, independente das características sociodemográ- ficas e de vínculo com a universidade. Essas associações não foram observadas em mulheres. Torna-se importante priorizar como questão de saúde pública o peso do comportamento sentado sobre os níveis de obesidade ${ }^{7}$. Especialmente em universitários, pois o regime de atividades acadêmicas que envolvem estudos na ins- 
tituição, normalmente na posição sentada pode assim contribuir com o aumento do IMC em níveis inadequados. Em termos gerais, o tempo sedentário acarreta diversos prejuízos à saúde dos universitários, seja com a obesidade ou outras comorbidades ${ }^{7}$. Deste modo, recorrer à implementação de pausas durante o elevado tempo exposto em posições que poupam energia representam ações fundamentais no período acadêmico.

Por outro lado, ao realizar as análises controlando também para os comportamentos relacionados à saúde (consumo de bebidas alcoólicas e consumo de carne vermelha com gordura), a associação entre tempo sentado e obesidade em homens não foi mantida. $\mathrm{O}$ consumo excessivo de álcool e o consumo de carne vermelha gordurosa em excesso estão diretamente associados ao excesso de peso em universitários ${ }^{10,26}$, e especialmente o consumo em excesso de carne vermelha está diretamente associado à obesidade ${ }^{27}$. $\mathrm{Na}$ medida em que, homens adultos jovens consomem bebida alcoólica em excesso, isso pode estimular o consumo de carne vermelha gordurosa, em virtude do contexto social de eventos desse grupo ${ }^{28}$. Deste modo, acredita-se que este fator explicaria os achados com universitários deste estudo, pois ambos os comportamentos confundiram a associação entre tempo sentado e obesidade.

Em relação à atividade física, as mulheres se destacaram na prática em níveis insuficientes, no entanto, tanto para elas quanto para os homens, neste estudo, não foram observadas associações em relação ao sobrepeso e a obesidade. Esta associação também não foi encontrada em universitários do curso de Medicina do Sudão ${ }^{20}$. Em um estudo com universitários colombianos, foram observadas prevalências de sobrepeso e obesidade de 20,26\% e 6,21\%, respectivamente, porém não houve associação com a atividade física ${ }^{29}$, Embora o IMC não permita distinguir massa magra e massa gor$\mathrm{da}$, o que neste caso pode apresentar resultados equivocados, quanto à classificação errônea de determinadas pessoas, especialmente aquelas envolvidas em práticas que podem favorecer o aumento da massa muscular, é inegável que em estudos epidemiológicos é um eficiente indicador de saúde ${ }^{29}$.

A prática de atividades físicas ${ }^{9}$ representa um importante comportamento para o controle do peso corporal para diferentes grupos populacionais com distintas faixas etárias e as recomendações apontam que é necessária à adoção de dose regular para essa manutenção ${ }^{9}$. Possivelmente, neste estudo, o desenho da pesquisa limita as inferências entre essas características, pois aqueles que estavam acima do peso, podem ter passado a ser mais ativos para a manutenção desse aumento de massa corporal, o que implicaria a inexistência de associação. Independente da não observação de diferenças em relação ao sobrepeso e a obesidade, os efeitos benéficos da atividade física devem ser priorizados nesse grupo, principalmente pelo contexto de tempo sentado que esse grupo é exposto e a outros componentes de riscos à saúde, como aqueles relacionados à ansiedade e o estresse.

Dentre as limitações deste estudo, pode-se caracterizar a obtenção da amostra por conveniência, pois é uma técnica de amostragem com maior fragilidade, por conta da propensão a participação de pessoas com melhores perfis de saúde. No entanto, para minimizar a tendência de participação de universitários de cursos específicos, a amostra foi estratificada de forma proporcional aos cursos, o que permitiu também a reposição das recusas dentro de cada curso. A utilização de questionário pode ser um limitador, por conta do viés de memória ${ }^{30}$, porém, as medidas deste estudo apresentam níveis satisfatórios de reprodutibilidade ${ }^{12}$ e de validade concorrente ${ }^{13,17}$. Medidas autorreferidas da massa corporal e estatura podem ser uma limitação, por conta do erro atribuído ao relato desses indicadores, porém, estudo mostra a consistência do uso dessas medidas em inquéritos epidemiológicos com universitários ${ }^{15}$. Possivelmente, alterações musculoesqueléticas de média a alta complexidade representam limitações quanto às informações referentes à prática de atividade física e comportamento sedentário, que não foram controladas no estudo.

Como pontos positivos, este estudo foi realizado com amostra representativa dos 25 cursos da sede da instituição. Soma-se a isso o período curto de coleta de dados que poderia sofrer influência do clima, feriados festivos e fim de semestre letivo, como maior ocorrência de atividades acadêmicas avaliativas.

As informações deste estudo poderão ser úteis para possíveis ações de intervenções com foco na saúde no âmbito universitário, especificamente relacionado à manutenção do peso corporal em universitários. Sugere-se que outros estudos possam estimar a associação da atividade física e do tempo sentado em relação ao sobrepeso e a obesidade, considerando o potencial papel dos indicadores sociodemográficos, de vínculo com a universidade e comportamentos relacionados à saúde como variáveis modificadoras de efeito, de forma melhor entender essas relações em universitários.

Conclui-se que a prevalência de sobrepeso foi maior 
em homens do que em mulheres. O tempo sentado foi associado à obesidade em homens, independente das características sociodemográficas e de vínculo com a universidade, porém não foi determinante após o controle dos comportamentos relativos à alimentação e bebidas alcoólicas. Não foi observada associação da atividade física em relação ao sobrepeso e a obesidade para ambos os sexos.

\section{Conflito de interesse}

Os autores declaram não haver conflito de interesse.

\section{Financiamento}

O presente trabalho foi realizado com apoio da Coordenação de Aperfeiçoamento de Pessoal de Nível Superior - Brasil (CAPES) - Código de Financiamento 001.

\section{Contribuição dos autores}

Barros GR, participou da concepção inicial do estudo, redação e revisão crítica do texto. Santos SFS, participou da concepção do manuscrito, revisão crítica do texto. Andaki ACR, participou da concepção do manuscrito, revisão crítica do texto. Sousa TF, participou da concepção inicial do estudo, orientação do artigo, redação e revisão crítica do texto.

\section{Agradecimentos}

Os autores agradecem a CAPES pela concessão da bolsa de mestrado ao primeiro autor (2018-2020); Ao Programa de Pós-Graduação em Educação Física da Universidade Federal do Triângulo Mineiro-UFTM, pois este estudo faz parte da dissertação intitulada: Excesso de peso corporal em universitários: prevalências e associação com atividade física e tempo sedentário.

\section{Referências}

1. Instituto Nacional de Estudos e Pesquisas Educacionais Anísio Teixeira. [acessado 2020 Jan 17]. Disponível em: http://portal.inep.gov.br/web/guest/censo-da-educacaosuperior

2. Winpenny EM, Smith M, Penney T, Foubister C, Guagliano JM, Love $\mathrm{R}$ et al. Changes in physical activity, diet, and body weight across the education and employment transitions of early adulthood: A systematic re-view and meta-analysis. Obes Rev. 2020;21(4):e12962.

3. Sousa TF, Barbosa AR. Prevalence of body weight excess in undergraduate students: analysis of repeated sur-veys. Rev Bras Epidemiol. 2017;20(4):586-97.

4. Li J, Simon G, Castro MR, Kumar V, Steinbach MS, Caraballo PJ. Association of BMI, comorbidities and allcause mortality by using a baseline mortality risk model. PLoS One. 2021;16(7):e0253696.

5. Associação Brasileira para Estudo da Obesidade e da Síndrome Metabólica. Diretrizes Brasileiras de Obesida-de, 2016. [acessado 2019 Jan 10]. Disponível em: http://www. abeso.org.br/diretrizes
6. Abdelaal M, le Roux CW, Docherty NG. Morbidity and mortality associated with obesity. Ann Transl Med. 2017;5(7):161.

7. Franco DC, Ferraz NL, Sousa TF. Sedentary behavior among university students: a systematic review. Rev Bras Cineantropom Desempenho Hum. 2019;21:e56485.

8. Tremblay MS, Aubert S, Barnes JD, Saunders TJ, Carson $\mathrm{V}$, Latimer-Cheung AE et al. Sedentary Behavior Research Network (SBRN) - Terminology Consensus Project process and outcome. Int J Behav Nutr Phys Act. 2017;14(1):75.

9. Brasil. Ministério da Saúde. Guia de Atividade Física para a População Brasileira. 2021. Disponível em: <https:// bvsms.saude.gov.br/bvs/publicacoes/guia_atividade_fisica_ populacao_brasileira.pdf $>$ [Agosto de 2021].

10. Cleland V, Schmidt M, Salmon J, Dywer T, Venn A. Combined associations of sitting time and physical activity with obesity in young adults. J Phys Act Health. 2014 ;11(1):136-44.

11. Luiz RR, Magnanini MMF. A lógica da determinação do tamanho da amostra em investigações epidemiológi-cas. Cad Saúde Colet. 2000;8(2):9-28.

12. Sousa TF, Fonseca SA, José HPM, Nahas MV. Validade e reprodutibilidade do questionário Indicadores de Saúde e Qualidade de Vida de Acadêmicos (Isaq-A). Arq Ciênc Esporte. 2013;1(1):21-30.

13. Matsudo S, Araújo T, Matsudo V, Andrade D, Andrade E, Oliveira LC, et al. Questionário Internacional de Atividade Física (IPAQ): estudo de validade e reprodutibilidade no Brasil. Rev Bras Ativ Fis Saúde. 2001;6(2):5-18.

14. World Health Organization. Obesity: preventing and managing the global epidemic: report of a WHO Consultation on Obesity, Geneva, 3-5 June 1997. 1997; Available in: https://apps.who.int/iris/handle/10665/63854 [2019 November].

15. Sousa TF, Barbosa AR. Validation of self-reported measures of body mass and stature in college students. ABCS Health Sci. 2016;41(2):71-7.

16. Owen N, Sparling PB, Healy GN, Dunstan DW, Matthews CE. Sedentary behavior: emerging evidence for a new health risk. Mayo Clin Proc. 2010;85(12):1138-41.

17. Franco DC, Farias GS, Pelegrini A, Virtuoso Jr. JS, Sousa TF. Validade das medidas do tempo sentado do questionário IPAQ-versão curta em universitários brasileiros. Rev Bras Ativ Fís Saúde 2021, no prelo.

18. Hallal PC, Victora CG, Wells JC, Lima RC. Physical inactivity: prevalence and associated variables in Brazili-an adults. Med Sci Sports Exerc. 2003;35(11):1894-900.

19. Morôco J. Análise de equações estruturais: fundamentos teóricos, software \& aplicações. 2a ed. Pêro Pinheiro: Cafilesa, 2014.

20. Yousif MM, Kaddam LA, Humeda HS. Correlation between physical activity, eating behavior and obesity among Sudanese medical students Sudan. BMC Nutr. 2019;5(6):2-8.

21. Sousa TF, José HPM, Barbosa AR. Condutas negativas à saúde em estudantes universitários brasileiros. Ciênc saúde coletiva. 2013;18(12):3563-75.

22. Sousa TF, Barbosa AR, Coelho F. Tempo de prática de atividade física no lazer como fator discriminatório da ausência de excesso de peso corporal. Rev Bras Ativ Fis Saúde. 2017;22(4):354-61.

23. Dawson DA. Defining risk drinking. Alcohol Res Health. 2011;34(2):144-56.

24. Alkazemi D. Gender differences in weight status, dietary habits, and health attitudes among college students in Kuwait: A cross-sectional study. Nutr Health. 2019;25(2):75-84. 
25. Sousa TF, Santos SFS, Rodrigues AS, Papini CB, Silva DAS, Tribess S. Prevalence and factors associated with body image dissatisfaction among university students: repeated surveys analysis. Rev Bras Cineantropom Desempenho Hum. 2019;21:e53036.

26. Nelson MC, Lust K, Story M, Ehlinger E. Alcohol use, eating patterns, and weight behaviors in a university population. Am J Health Behav. 2009;33(3):227-37.

27. Schlesinger S, Neuenschwander M, Schwedhelm C, Hoffmann G, Bechthold A, Boeing H, et al. Food groups and risk of overweight, obesity, and weight gain: a systematic review and dose-response meta-analysis of pro-spective studies. Adv Nutr. 2019;10(2):205-218.

28. Longo-Silva G, Silveira JAC, Menezes RCE, Marinho PM, Epifânio SBO, Brebal KMM, et al. Tendência temporal e fatores associados ao consumo de carnes gordurosas na população brasileira entre de 2007 a 2014. Ciênc saúde coletiva. 2019;24(3):1175-88.
29. Rangel Caballero LG, Sánchez LZR, Delgado EMG. Sobrepeso y obesidad en estudiantes universitarios colombianos y su asociación con la actividad física. Nutr Hosp. 2015;31(2):629-36.

30. Hallal PC, Dumith SC, Bastos JP, Reichert FF, Siqueira FV, Azevedo MR. Evolução da pesquisa epidemioló-gica em atividade física no Brasil: revisão sistemática. Rev. Saúde Pública. 2007;41(3):453-60.

Recebido: $14 / 04 / 2021$

Aprovado: 27/09/2021

Como citar este artigo:

Barros GR, Santos SFS, Andaki ACR, Sousa TF. Sobrepeso e obesidade em universitários: prevalências e fatores associados. Rev Bras Ativ Fís Saúde. 2021;26:e0225. DOI: 10.12820/rbafs.26e0225 